\title{
Quantitative computed tomography texture analysis: can it improve diagnostic accuracy to differentiate malignant lymph nodes?
}

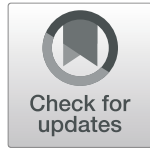

So Youn Shin ${ }^{1}$, II Ki Hong ${ }^{2}$ and Yong Suk Jo ${ }^{3,4^{*}}$ (i)

\begin{abstract}
Background and objective: Mediastinal lymph node (LN) staging in individuals with non-small-cell lung cancer plays an important role in staging and treatment planning. This study aimed to assess the accuracy of computed tomography $(\mathrm{CT})$ texture analysis (CTTA) in differentiating benign and malignant mediastinal LNs.

Methods: Pathologically confirmed malignant and benign mediastinal LN samples, obtained using endobronchial ultrasound-guided transbronchial needle aspiration (EBUS-TBNA), were retrospectively reviewed, in addition to chest CT and 18-fluorodeoxyglucose (FDG) uptake positron emission tomography (PET) data. For each LN, CTTA was performed using "AVIEW" software (Coreline Soft, Republic of Korea) by drawing a region of interest.

Results: A total of 132 LNs from 80 patients were included and classified into two groups according to pathology results: malignant $(n=61)$ and benign $(n=71)$. In EBUS, size $>1 \mathrm{~cm}$, round shape, heterogeneous echogenicity, and presence of coagulation necrosis sign were more prevalent in malignant than in benign LNs; length was the only feature that distinguished the two groups. Among CTTA features, compactness and normalized standard deviation (SD) showed differences between the two groups. The ability to distinguish malignant LNs was higher using high standard uptake value (SUV) on FDG PET/CT (SUV $\max \geq 5$ ) and normalized SD on CTTA (area under the receiver operating characteristic curve 0.739 versus 0.742 , respectively); however, normalized SD demonstrated very low sensitivity despite high specificity.
\end{abstract}

Conclusions: CTTA may be helpful in distinguishing between benign and malignant LNs; however, the diagnostic value was not high. Therefore, integrated evaluation with other imaging modalities is needed.

Keywords: Texture and shape analysis, Mediastinal lymph node, Malignancy

\section{Background}

Endobronchial ultrasound-guided (EBUS) transbronchial needle aspiration (EBUS-TBNA) is a diagnostic method used to evaluate mediastinal lymphadenopathies, including lung cancer and non-malignant diseases, such as tuberculosis and sarcoidosis [1-4]. The diagnostic yield of EBUS-TBNA is reported to be 93-97\% [4], which is comparable with that of surgical mediastinoscopy; however, EBUS-TBNA has some advantages over mediastinoscopy.

\footnotetext{
* Correspondence: lucidyonge@gmail.com

${ }^{3}$ Division of Pulmonary and Critical Care Medicine, Department of Internal Medicine, KyungHee University Hospital, Seoul, Republic of Korea

${ }^{4}$ Department of Internal Medicine, Division of Pulmonary, Allergy, and Critical Care Medicine, Hallym University Kangdong Sacred Heart Hospital, Seoul, Republic of Korea

Full list of author information is available at the end of the article
}

EBUS-TBNA is a minimally invasive procedure, and it is possible to approach hilar/interlobar lymph nodes (LNs) and retrocardiac LNs that cannot be accessed using mediastinoscopy [5].

According to guidelines addressing mediastinal staging in lung cancer, discrete $\mathrm{LN}(\mathrm{s})$ on chest computed tomography (CT) with high 18F-fluorodeoxyglucose (FDG) uptake on positron emission tomography (PET) should be visualized for accurate staging [6]. However, these findings do not necessarily correspond with cytopathological analysis, thus resulting in false-positive results $[3,7]$. To overcome this barrier, quantitative CT texture analysis (CTTA) was developed to analyze various imaging findings of the LN itself using specialized software, which provides information

(C) The Author(s). 2019 Open Access This article is distributed under the terms of the Creative Commons Attribution 4.0 International License (http://creativecommons.org/licenses/by/4.0/), which permits unrestricted use, distribution, and reproduction in any medium, provided you give appropriate credit to the original author(s) and the source, provide a link to the Creative Commons license, and indicate if changes were made. The Creative Commons Public Domain Dedication waiver (http://creativecommons.org/publicdomain/zero/1.0/) applies to the data made available in this article, unless otherwise stated. 
beyond that obtained by morphological and size assessment performed using the naked eye.

It has been reported that sonographic features can be useful imaging tools for the evaluation of mediastinal and hilar LNs during EBUS [8-12]. Schmid-Bindert et al. [8] suggested six features including the following: short axis length; heterogeneous pattern; round shape; distinct margin; absence of central hilar structure; and high blood flow. In addition, they reported that heterogeneity was the most powerful feature for prediction of malignant LNs. More recently, Fujiwara et al. [9] suggested four items indicating malignant LNs: round shape, distinct margin, heterogeneous echogenicity, and coagulation necrosis sign (CNS). None of these features were present in $96 \%$ of patients, who were determined to have no malignancy.

In typical clinical settings, imaging techniques, such as chest CT and/or PET, are performed before EBUS-TBNA. Thus, detailed assessment of LNs using imaging and ultrasound evaluation based on these findings may contribute to diagnostic accuracy. The purpose of the present study, therefore, was to identify features that help distinguish benign and malignant LNs from mediastinal and/or hilar LNs explored using EBUS-TBNA, and to assess the utility of CTTA in this regard.

\section{Methods}

\section{Patients}

A retrospective review of medical records was performed among patients who underwent EBUS-TBNA for evaluation of mediastinal and/or hilar LN(s), either for staging of lung cancer or lymphadenopathy alone, at the Department of Pulmonology, KyungHee University Hospital, (KHUH, Seoul, Korea) between March 2017 and July 2018. EBUS-TBNA was performed for patients with radiologically identified mediastinal and/or hilar LNs on enhanced chest CT imaging. $\mathrm{LN}$ stations were determined according to the International Association for the Study of Lung Cancer (IASLC) guideline [13]. Cases with only primary mass punctures were excluded from this study. LNs classified as benign or malignant according to pathological results were included in the analysis according to chest CT, PET/CT, CTTA, and EBUS features (Fig. 1). The present study was approved by the Ethics Review Board of KHUH (IRB No., KHUH 201808-023).

\section{EBUS-TBNA}

EBUS-TBNA was performed on an inpatient basis with patients under conscious sedation (midazolam and fentanyl) using local anesthesia with lidocaine. Midazolam is routinely infused at $0.05 \mathrm{mg} / \mathrm{kg}$, and fentanyl $(25 \mathrm{mcg})$

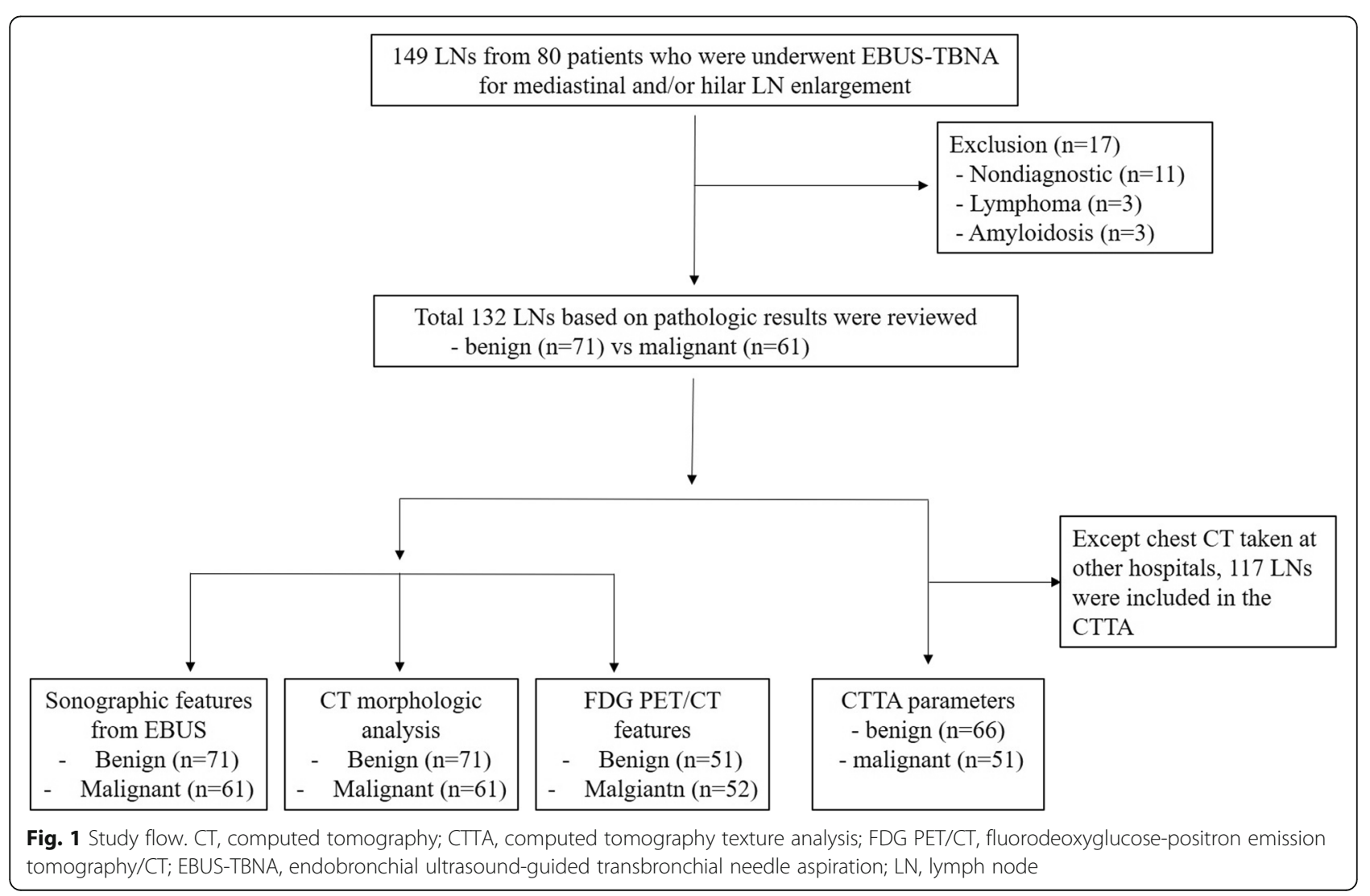


was administered before commencing EBUS-TBNA. If sedation was insufficient or the patient was irritable or awake during the procedure, an additional 1-2 mg dose of midazolam was administered. A real-time linear probe (BF-UC260FW; Olympus, Tokyo, Japan) was used for EBUS and a 22-gauge needle (NA-201SX-4022; Olympus) for TBNA. After white-light bronchoscopic evaluation, the EBUS scope, equipped with a convex probe, was inserted. LN location was determined based on the standard LN map, and all procedures were performed by a single expert bronchoscopist. The number of punctures and decisions regarding procedure completion were at the discretion of the operator according to the clinical situation.

\section{EBUS image characteristics of LNs}

All EBUS images were assessed by a single attending pulmonologist, who performed all EBUS-TBNA in this study immediately after completion of the procedure in the bronchoscopy room. LN features were categorized based on the EBUS images [8, 9]: short axis diameter $(<1 \mathrm{~cm}$ or $\geq 1 \mathrm{~cm})$; shape (round or oval); margin (distinct or indistinct); echogenicity (homogeneous or heterogeneous); central hilar structure (absent or present); and CNS (absent or present) (See Additional file 1 Figure S1).

\section{CT scanning protocol and CT image analysis}

Chest CT images were acquired using 128-detector (Ingenuity Core, Philips Healthcare), 64-detector (Brilliance, Philips Healthcare), or 160-detector (Aquillion Prime, Toshiba Healthcare) scanners. Scan parameters included the following: power, $120 \mathrm{kV}$; and current, 100-400 effective $\mathrm{mA}$ with dose modulation. Reconstruction intervals were $2 \mathrm{~mm}$ thick, which is a $2 \mathrm{~mm}$ interval without a gap. Enhanced CT images were acquired using a 45-s delay after intravenous administration of $100 \mathrm{~mL}$ noninonic contrast medium (hexosure [iohexol], LG, South Korea) and $50 \mathrm{~mL}$ of normal saline at a rate of $2.4 \mathrm{~mL} / \mathrm{s}$ using a power injector. All images were viewed on the mediastinal (window width, 450 Hounsfield unit [HU]; window level, $50 \mathrm{HU}$ ) and lung window (window width, $1500 \mathrm{HU}$; window level, $-700 \mathrm{HU}$ ) settings on the axial and coronal images on the picture archiving communicating system (PACS).

An experienced thoracic radiologist retrospectively reviewed all $\mathrm{CT}$ images. $\mathrm{CT}$ images were analyzed using a slice thickness as thin as possible (CT images with 2-mm slice thickness were acquired in the authors' hospital [135 LNs from 68 patients]; CT images with $2.5-\mathrm{mm}$ to $5-\mathrm{mm}$ slice thickness were acquired at other hospitals [14 LNs from 12 patients]). For CTTA, CT images acquired at the authors' hospital were analyzed, except images acquired at other hospital.

Using axial and coronal CT scans, the following morphological features were assessed: size (the maximal short- and long-axis diameters); shape (oval or round); margin (well-defined or ill-defined); presence of internal components, such as calcification, fat, or necrosis; and nodal density (HU). The location of the LN was also described: right, left or subcarina, and ipsilateral or contralateral, according to the primary lung cancer.

\section{Quantitative CTTA}

CTTA was performed using commercially available software (AVIEW Research, Coreline Soft, Republic of Korea) by drawing a region of interest (ROI) on a single image with the maximal nodule dimension in the axial plane (Fig. 2). ROIs with calcifications, vessels, and areas related to beam hardening artifact(s) were excluded from the ROIs. After drawing the ROI on an axial image, the following histogram features were obtained from the LNs: mean attenuation; standard deviation (SD); skewness; kurtosis; entropy; and CT attenuation values at the 10th, 25th, 50th, 75th, 90th, and 95th percentiles. Skewness refers to asymmetry of the histogram and calculated as $\mathrm{E}\left[\left(\frac{X-\mu}{\sigma}\right)^{3}\right]$, in which $\mathrm{X}=$ attenuation, $\sigma=$ mean of attenuation, and $\mu=$ standard deviation of attenuation. Kurtosis means the magnitude of the distribution and calculated as $\mathrm{k}$ $=\frac{E(x-\mu)^{4}}{\sigma^{4}}-3$, in which $\mathrm{x}=$ attenuation, $\sigma=$ mean of attenuation, and $\mu=$ standard deviation of attenuation. Entropy refers to the irregularity or complexity of the lesion, and calculated as $\sum_{i=1}^{n} p(x i) \log p(x i)$, in which $x_{i}=$ frequency from histogram of ROI and $p\left(x_{i}\right)=$ probability on histogram $[14,15]$. SD can be affected by size, especially in small lesions; thus, size normalized SD [16] (nSD: SD/N [total number of pixels within the ROI]) was additionally analyzed, which calculated as $\frac{\operatorname{Ln}(S D)}{\operatorname{Ln}(N)}$.

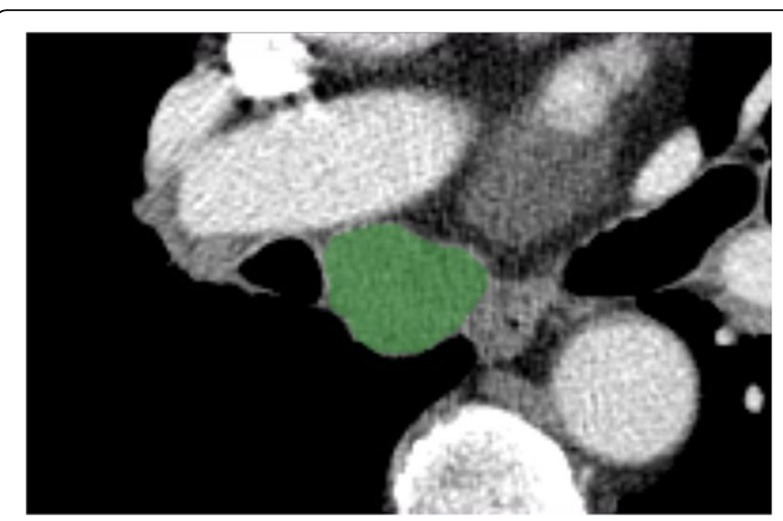

Fig. 2 Representative computed tomography image illustrating a region of interest $(\mathrm{ROI})$ demonstrating the enlarged subcarinal lymph node. The green highlighted area represents the ROI 
${ }^{18} \mathrm{FDG}$ PET/CT acquisition protocol

${ }^{18}$ F-FDG PET/CT was performed using a Gemini TF16 PET scanner (Philips Healthcare, Cleveland, $\mathrm{OH}$ ), which has an intrinsic resolution of $4.8 \mathrm{~mm}$ full-width at half-maximum and simultaneous imaging of 50 contiguous transverse planes with a thickness of $4 \mathrm{~mm}$ for a longitudinal field of view of $18 \mathrm{~cm}$. All patients fasted for at least $6 \mathrm{~h}$ before PET imaging. Imaging was initiated using a planar scout scan to define the axial range of the study, followed by volumetric CT acquisition. CT parameters were $120 \mathrm{kVp}, 50 \mathrm{mAs}$, and 2-mm slice width and separation. A 1-min emission scan per bed position was performed after the intravenous injection of ${ }^{18} \mathrm{~F}$-FDG of $296 \mathrm{MBq}$. All emission scans were performed in three-dimensional acquisition mode. Attenuation-corrected images were reconstructed into a $144 \times 144$ matrix by means of an ordered subset expectation maximization algorithm incorporating TOF information (TOF-OSEM). The reconstruction parameters for TOF-OSEM were 3 iterations and 33 subsets.

\section{FDG PET/CT image analysis}

FDG PET/CT is the most widely used non-invasive diagnostic modality for detection of primary tumor(s) and metastasis [17]. Maximum and peak standardized uptake value (SUV) and $\mathrm{HU}$ of the LNs were measured using MIRADA software using ROI covering the LNs. SUVs alone and SUVmax $\geq 2.5$ and $\mathrm{HU}<70$ were used as the criteria to diagnose LNs [18].

\section{Statistical analysis}

Pearson's chi-squared test for categorical variables, and the Student's t-test or Fisher's exact test for continuous variables, were used to compare baseline characteristics, and are expressed as absolute number with percentages, or mean \pm standard deviation, unless otherwise indicated. All LNs were categorized according to the final pathology report. To determine which features of various imaging modalities, including chest $\mathrm{CT}$, quantitative CTTA, PET/CT and EBUS, were the most relevant to distinguish benign and malignant LNs, area under the receiver operating curve (AUROC) analysis was performed. A classification and regression tree (CART) analysis-a type of decision-tree methodology for the identification of mutually exclusive subgroups of at-risk individuals who share common characteristics related to particular health-related behavior-was also performed. Sensitivity and specificity were calculated for various radiological features.

All analyses were two-sided, and $P<0.05$ was considered to be statistically significant. All analyses were performed using STATA version 14.2 (StataCorp, College Station, TX, USA).
Table 1 Patient characteristics

\begin{tabular}{|c|c|}
\hline Characteristic & Value \\
\hline Patients, n & 80 \\
\hline Age (years) & $69(60-77)$ \\
\hline Male sex & $53(66.3)$ \\
\hline \multicolumn{2}{|l|}{ Smoking status } \\
\hline Never smoker & $24(30.0)$ \\
\hline Ex-smoker & $32(40.0)$ \\
\hline Current smoker & $24(30.0)$ \\
\hline Smoking pack-year & $40(25-50)$ \\
\hline \multicolumn{2}{|l|}{ Underlying condition } \\
\hline \multicolumn{2}{|l|}{ Previous malignancy } \\
\hline NSCLC & $7(8.8)$ \\
\hline Extra-thoracic malignancy & $11(13.8)$ \\
\hline Lymphoma & $3(3.8)$ \\
\hline Interstitial lung disease & $2(2.5)$ \\
\hline Previous history of pulmonary tuberculosis & $4(5.0)$ \\
\hline COPD & $18(22.5)$ \\
\hline \multicolumn{2}{|l|}{ Purpose of EBUS-TBNA } \\
\hline Lung cancer staging and/or diagnosis & $65(81.3)$ \\
\hline Metastasis from extra-thoracic malignancy & $10(12.5)$ \\
\hline Lymphoma & $2(2.5)$ \\
\hline Sarcoidosis & $3(3.7)$ \\
\hline Examined lymph node, total & 149 \\
\hline Examined lymph node per patients & $2(1-3)$ \\
\hline \multicolumn{2}{|l|}{ Nodal station ${ }^{*}$} \\
\hline $2 \mathrm{R}$ & $14(9.4)$ \\
\hline $2 \mathrm{~L}$ & $1(0.7)$ \\
\hline $4 \mathrm{R}$ & $41(27.5)$ \\
\hline $4 \mathrm{~L}$ & $18(12.1)$ \\
\hline 7 & $47(31.5)$ \\
\hline $10 \mathrm{R}$ & $9(6.0)$ \\
\hline $10 \mathrm{~L}$ & $9(6.0)$ \\
\hline $11 R$ & $5(3.4)$ \\
\hline $11 \mathrm{~L}$ & $2(1.3)$ \\
\hline 3P: paraesophageal & $2(1.4): 1(0.7)$ \\
\hline$S U V_{\max }$ on FDG PET/CT ${ }^{\dagger}$ & $5.0(2.3-6.3)$ \\
\hline
\end{tabular}

Data presented as $n$ (\%) or median (interquartile range), unless otherwise indicated

${ }^{*} 2 \mathrm{R}$, right upper paratracheal; $2 \mathrm{~L}$, left upper paratracheal; $4 \mathrm{R}$, right lower paratrachea; $4 \mathrm{~L}$, left lower paratracheal; 7 , subcarina; 10R, right hilar; $10 \mathrm{~L}$, left hilar; $11 \mathrm{R}$, right interlobar; $11 \mathrm{~L}$, left interlobar; $3 \mathrm{P}$, retrotracheal

${ }^{\dagger} 18$-Fluorodeoxyglucose positron emission tomography/computed tomography (FDG PET/CT) results were available from 103 lymph nodes in 57 patients

COPD, chronic obstructive pulmonary disease; EBUS-TBNA, endobronchial ultrasound-guided transbronchial needle aspiration; NSCLC, non-small cell lung cancer; SUV max maximal standardized uptake value 


\section{Results}

\section{Patient characteristics}

Among 80 patients who underwent EBUS-TBNA for mediastinal and/or hilar LN enlargement, 149 LNs were examined. The mean age of the cohort was 69 years, males were predominant $(n=53[66.3 \%])$, and former smokers accounted for $70 \%$, with a mean history of 40 pack-years. The most common purpose of the EBUS-TBNA procedure was to diagnose or stage lung cancer. A mean of 2 LNs were punctured per patient; subcarinal (7) and right lower paratracheal (4R) LNs were obtained in 31.5 and $27.5 \%$ of cases, respectively, and left lower paratracheal $(4 \mathrm{~L})$, right upper paratracheal (2R), right hilar (10R) and left hilar (10 L) LNs were followed (Table 1).

The final diagnoses according to cytological and histological results are summarized in Table 2. Lung cancers, including non-small cell lung cancer (NSCLC) and small cell lung cancer, were diagnosed in $35.5 \%$ of cases, while reactive hyperplasia was diagnosed in 36.2\%. Among 149 LNs, 13 were classified as nondiagnostic due to the presence of a bloody smear only or paucicellularity. After exclusion of cases involving lymphoma, amyloidosis and nondiagnostic specimens, 132 LNs were classified into two groups: malignant $(n=61)$ and benign $(n=71)$.

\section{Sonographic findings of EBUS}

A short-axis diameter, measured from the ultrasound reference line as $>1 \mathrm{~cm}$, was more frequently found in

Table 2 Final diagnosis according to cytological and pathological results of lymph nodes $(n=149)$

\begin{tabular}{ll}
\hline Diagnosis & $\mathrm{n}(\%)$ \\
\hline Lung cancer & \\
$\quad$ NSCLC & $53(35.5)$ \\
$\quad$ Squamous cell carcinoma & $16(10.7)$ \\
$\quad$ Adenocarcinoma & $20(13.4)$ \\
$\quad$ SCLC & $16(10.7)$ \\
$\quad$ Neuroendocrine carcinoma & $1(0.7)$ \\
Metastasis from extrathoracic malignancy & $6(4.0)$ \\
Lymphoma & $3(2.0)$ \\
Tuberculosis & $1(0.7)$ \\
Sarcoidosis & $4(2.7)$ \\
Amyloidosis & $3(2.0)$ \\
Reactive hyperplasia & $54(36.2)$ \\
Nonspecific inflammation & $5(3.4)$ \\
Fungal infection & $1(0.7)$ \\
Necrosis & $4(2.7)$ \\
Leiomyoma & $2(1.3)$ \\
Non-diagnostic & $13(8.9)$ \\
\hline NSCLC Non-smal
\end{tabular}

NSCLC, Non-small cell lung cancer; SCLC, Small cell lung cancer malignant than in benign LNs $(57.8 \%$ versus [vs] $88.5 \%$; $P<0.001)$. A round shape and heterogeneous echogenicity were more common in malignant LNs $(39.4 \%$ vs 59.0 and $11.3 \%$ vs $37.7 \% ; P<0.05$ for both). CNS was also encountered more frequently in malignant LNs (1.4\% vs $9.8 \% ; P=0.031$ ) (Table 3 ).

\section{Two-dimensional and textural features of chest $\mathrm{CT}$}

Various chest CT parameters and CT textural features are summarized in Table 4. Seventy-one benign and 61 malignant LNs were included in the CT parameter analysis. LNs located in the same direction as the primary tumor were more prevalent in malignant LNs and, those in the opposite direction were more prevalent in benign LNs; however, the difference was not statistically significant. It was not possible to assess the position of 13 benign and 7 malignant LNs due to sarcoidosis and tuberculosis lymphadenopathy involved in the $\mathrm{LN}$ alone. Short-axis diameter was longer in malignant compared with benign LNs $(15.7 \mathrm{~mm}$ vs $11.2 \mathrm{~mm}$, respectively; $P<$ 0.001). Shape, well-demarcated margin, and HU between benign and malignant LN were not significantly different.

A total of 117 LNs were included in CTTA, except CT, which included only non-contrast images acquired at external hospitals with low quality that did not match the chest CT at KHUH. CTTA was evaluated in 66 benign and 51 malignant LNs. Compactness and normalized SD were significantly different between the benign and malignant LNs $(P<0.001$ for both). There were no significant differences noted in the remaining CTTA parameters including mean $\mathrm{HU}$, entropy, skewness, and kurtosis.

Table 3 Sonographic features of benign and malignant lymph nodes (LNs)

\begin{tabular}{|c|c|c|c|}
\hline Characteristic & Benign & Malignant & $P$ value \\
\hline Included lymph nodes & $71(53.8)$ & $61(46.2)$ & \\
\hline \multicolumn{4}{|l|}{ Sonographic features } \\
\hline Size, $\geq 1 \mathrm{~cm}$ & $41(57.8)$ & $54(88.5)$ & $<0.0001$ \\
\hline Shape: round & $28(39.4)$ & $36(59.0)$ & 0.025 \\
\hline Margin, distinct & $67(94.4)$ & $60(98.4)$ & 0.231 \\
\hline \multicolumn{4}{|l|}{ Echogenicity: } \\
\hline Homogeneous & $52(88.7)$ & $38(62.3)$ & $<0.0001$ \\
\hline Heterogeneous & $8(11.3)$ & $23(37.7)$ & \\
\hline Central hilar structure, present & $48(67.6)$ & $32(52.5)$ & 0.076 \\
\hline Coagulation necrosis sign, present & $1(1.4)$ & $6(9.8)$ & 0.031 \\
\hline
\end{tabular}


Table 4 Morphological computed tomography (CT), CT texture analysis (CTTA), and 18-fluorodeoxyglucose positron emission tomography/CT (FDG PET/CT) parameters for malignant and benign lymph nodes

\begin{tabular}{|c|c|c|c|}
\hline & Benign & Malignant & $P$ value \\
\hline Morphological CT parameters, including lymph nodes, $\mathrm{n}$ & 71 & 61 & \\
\hline \multicolumn{4}{|l|}{ Location of primary tumor and lymph node } \\
\hline Ipsilateral & $25(50.7)$ & $40(65.6)$ & 0.384 \\
\hline Contralateral & $19(26.8)$ & $12(19.7)$ & \\
\hline Subcarinal & $3(4.2)$ & $2(3.3)$ & \\
\hline Short axis diameter, mm & $11.2 \pm 4.2$ & $15.7 \pm 6.3$ & $<0.0001$ \\
\hline Long axis diameter, mm & $16.2 \pm 5.2$ & $22.2 \pm 7.6$ & $<0.0001$ \\
\hline Shape, round & $7(9.9)$ & $10(16.4)$ & 0.264 \\
\hline Margin, well-defined & $64(90.1)$ & $52(85.3)$ & 0.390 \\
\hline Noticeability (> $60 \mathrm{HU})$ & $52(73.2)$ & $38(62.3)$ & 0.178 \\
\hline $\mathrm{CT}$ texture parameters, including lymph nodes & 66 & 51 & \\
\hline Area $\left(\mathrm{mm}^{2}\right)$ & $160.3 \pm 111.1$ & $338.8 \pm 263.5$ & $<0.0001$ \\
\hline Circularity & $0.84 \pm 0.07$ & $0.83 \pm 0.07$ & 0.358 \\
\hline Compactness & $0.97 \pm 0.01$ & $0.98 \pm 0.01$ & 0.003 \\
\hline Roundness & $0.76 \pm 0.08$ & $0.77 \pm 0.07$ & 0.544 \\
\hline Entropy & $6.6 \pm 0.5$ & $6.8 \pm 0.5$ & 0.142 \\
\hline Mean HU & $69.6 \pm 23.5$ & $68.3 \pm 23.0$ & 0.774 \\
\hline Standard deviation (SD) & $43.2 \pm 19.6$ & $38.0 \pm 13.6$ & 0.108 \\
\hline Normalized SD* & $0.19 \pm 0.13$ & $0.10 \pm 0.08$ & $<0.0001$ \\
\hline Skewness & $-0.00 \pm 0.02$ & $0.00 \pm 0.02$ & 0.562 \\
\hline Kurtosis & $3.1 \pm 0.7$ & $3.2 \pm 0.9$ & 0.914 \\
\hline 10th percentile HU & $11.8 \pm 31.9$ & $19.3 \pm 26.3$ & 0.174 \\
\hline 25th percentile HU & $41.2 \pm 24.7$ & $42.2 \pm 23.2$ & 0.816 \\
\hline 50th percentile HU & $71.7 \pm 23.0$ & $68.1 \pm 23.2$ & 0.397 \\
\hline 75th percentile $\mathrm{HU}$ & $101.0 \pm 28.0$ & $94.4 \pm 26.4$ & 0.194 \\
\hline 90th percentile HU & $125.0 \pm 36.3$ & $116.4 \pm 32.1$ & 0.185 \\
\hline FDG PET/CT parameter, Included lymph nodes & 51 & 52 & \\
\hline$S U V_{\max }$ & $3.4 \pm 2.4$ & $6.3 \pm 3.8$ & $<0.0001$ \\
\hline SUV Peak & $2.7 \pm 1.6$ & $5.2 \pm 3.1$ & $<0.0001$ \\
\hline Maximal HU $\mathrm{HU}^{\dagger}$ & $119.1 \pm 283.5$ & $80.4 \pm 147.3$ & 0.385 \\
\hline$S U V_{\max } \geq 2.5$ and $\mathrm{HU}<70$ & $15(29.4)$ & $40(76.9)$ & $<0.0001$ \\
\hline
\end{tabular}

Data presented as $\mathrm{n}(\%)$ or mean \pm standard deviation, unless otherwise indicated

*Normalized standard deviation (SD) is calculated as $\ln (S D) / \ln (n)[=\ln (S D) / \ln ($ total number of pixels within the region of interest)]

$H U$, Hounsfield unit; SUV, standard uptake value

${ }^{+} \mathrm{HU}$ values obtained from non-enhanced chest $C T$ performed with FDG PET/CT

\section{FDG PET/CT results}

Maximum and peak SUV were significantly higher in malignant than in benign LNs (6.3 vs 3.4 ; and 5.2 vs 2.7 , respectively; $P<0.001$ for both). Although maximal HU was higher in benign LNs, the difference was not statistically significant (Table 4). Peak SUV value $\geq 4$ and maximal SUV value $\geq 5$ were good determinants of malignant LNs in the CART analysis (relative hazard risk, 1.70 and 1.69, respectively). The combined PET/CT parameters of SUV$\max \geq 2.5$ and $H U<70$ was significantly higher in malignant than benign LNs (76.9\% vs $29.4 \%$, respectively).

\section{Diagnostic yield of the imaging modalities}

The diagnostic accuracy and sensitivity of various features extracted from chest CT, EBUS, CTTA and PET/ CT are shown in Table 5. ROC analyses revealed that normalized SD demonstrated the highest accuracy for differentiating benign and malignant LNs when benign was encoded as 1 and malignant $\mathrm{LN}$ as 0 , unlike other parameters (AUC, 0.742). High maximal SUV $(\geq 5)$ and high peak SUV $(\geq 4)$ on PET/CT demonstrated similar ability to distinguish benign and malignant LNs (AUC, 0.739 for both), followed by compactness on CTTA 
Table 5 Receiver operating characteristic curve analysis of chest computed tomography (CT), textural features, 18fluorodeoxyglucose positron emission tomography/CT (FDG PET/CT) and sonographic findings

\begin{tabular}{|c|c|c|c|c|c|}
\hline Variable & AUC & SE (AUC) & $95 \% \mathrm{Cl}$ & Se $(\%)$ & $\mathrm{Sp}(\%)$ \\
\hline Short-axis diameter, $\geq 10 \mathrm{~mm}$ & 0.659 & 0.038 & $0.585-0.732$ & 93 & 58.7 \\
\hline Compactness on CTTA & 0.666 & 0.051 & $0.566-0.766$ & 10.6 & 100 \\
\hline Normalized SD on $\mathrm{CTTA}^{+}$ & 0.742 & 0.046 & $0.652-0.833$ & 8.2 & 100 \\
\hline \multicolumn{6}{|l|}{ FDG PET/CT } \\
\hline SUV $V_{\text {max }} \geq 2.5$ & 0.639 & 0.042 & $0.556-0.721$ & 94.4 & 55.8 \\
\hline$S U V_{\max } \geq 5$ & 0.739 & 0.042 & $0.657-0.821$ & 74.7 & 94.3 \\
\hline SUV $V_{\text {Peak }} \geq 4$ & 0.739 & 0.041 & $0.658-0.820$ & 73 & 95.6 \\
\hline$S U V_{\max } \geq 2.5$ and $\mathrm{HU}<70$ & 0.738 & 0.044 & $0.652-0.823$ & 87.5 & 82.5 \\
\hline Size on EBUS, $\geq 10 \mathrm{~mm}$ & 0.654 & 0.036 & $0.583-0.724$ & 95.3 & 54.6 \\
\hline Round shape & 0.598 & 0.043 & $0.513-0.682$ & 71.4 & 72 \\
\hline Heterogeneous echogenicity & 0.632 & 0.037 & $0.561-0.704$ & 51 & 95 \\
\hline Coagulation necrosis sign & 0.542 & 0.021 & $0.502-0.582$ & 20.2 & 100 \\
\hline
\end{tabular}

AUC, Area under the receiver operating characteristic curve; $C l$, Confidence interval; EBUS, Endobronchial ultrasound; SD, Standard deviation; Se, Sensitivity; Sp, Specificity; SUVmax, Standard maximal uptake value

${ }^{\dagger}$ Analyses was performed using $1=$ benign and $0=$ malignant

(AUC, 0.666), short axis diameter $>10 \mathrm{~mm}$ on chest CT and EBUS (AUC, 0.659 and 0.654, respectively), and heterogenic echogenicity (AUC, 0.632).

However, compactness and normalized SD on CTTA had very low sensitivity, despite high specificity. LN size demonstrated low specificity and, echogenic characteristics, such as echogenicity and the presence of CNS, had relatively low sensitivity. Only PET/CT criteria combining $\mathrm{SUV}_{\max } \geq 2.5$ and $\mathrm{HU}<70$ had high sensitivity $(87.5 \%)$ and specificity $(82.5 \%)$ for malignancy.

\section{Diagnostic yield of combining features of chest $\mathrm{CT}$ and $\mathrm{PET} / \mathrm{CT}$}

Diagnostic accuracy and sensitivity analyses were also performed by combining three two-dimensional chest CT and CTTA features (short-axis diameter $\geq 10 \mathrm{~mm}$, compactness and normalized SD) and that of PET/CT as well $\left(\mathrm{SUV}_{\max } \geq 2.5\right.$ and $\mathrm{HU}<70, \mathrm{SUV}_{\text {max }} \geq 5$ and SUVpeak $\geq 4$ ) (Additional file 2 Table S1). Several combinations of each item of chest CT and CTTA did not achieve better diagnostic accuracy. The combination of CT features and SUV, either maximal or peak value, also did not yield better accuracy, and only size on two-dimensional chest CT and the combination of SUV$\max \geq 2.5$ and $\mathrm{HU}<70$ demonstrated slightly higher diagnostic accuracy.

\section{Discussion}

We analyzed imaging features of various modalities, including chest CT, PET/CT, CTTA and EBUS, used to distinguish malignant and benign LNs, which were pathologically identified using EBUS-TBNA, and evaluated their diagnostic accuracy. In addition to established findings in malignant LNs, such as size, shape, echogenicity, CNS in EBUS, size on chest CT, high SUV in PET/CT, compactness, and normalized SD on CTTA were features that distinguished malignant and benign LNs. Although normalized SD on CTTA demonstrated high diagnostic accuracy, the sensitivity was low, which suggests it should not be used as a sole diagnostic feature.

Mediastinal LN staging plays a pivotal role in staging and treatment planning in lung cancer. Traditionally, chest $\mathrm{CT}$ and PET/CT have been used to predict malignancy. However, a meta-analysis comparing the accuracy of PET/CT and chest CT in detecting mediastinal LN metastases in patients with NSCLC reported that PET/ $\mathrm{CT}$ was more accurate than chest $\mathrm{CT}$ in distinguishing metastatic mediastinal LNs [7]. However, there is the possibility of false-positive results when evaluating only FDG uptake on PET/CT, and it has been reported that it is more likely to be benign LN, with higher attenuation, than surrounding vessels or calcified LNs $[19,20]$. Lee et al. [21] reported that integrated SUVmax and LN density criteria demonstrated higher accuracy for characterizing metastatic NSCLC. We compared the criteria based on SUVmax $\geq 2.5$, widely known as a positive standard [22, 23 ], and $\geq 5$ with high discriminatory power in our study. Criteria combining SUVmax $\geq 2.5$ and $\mathrm{HU}<70$ [18] in our study revealed that combined PET/CT criteria demonstrated higher diagnostic accuracy than SUVmax alone, and similar accuracy with higher SUVmax $(\geq 5)$. Nevertheless, a Cochrane systematic review, including 45 studies that assessed the diagnostic accuracy of PET/ $\mathrm{CT}$ for diagnosing N2 disease in patients with resectable NSCLC, reported sensitivity and specificity estimates for the SUVmax $\geq 2.5 \mathrm{PET} / \mathrm{CT}$ positivity criteria were $81.3 \%$ (95\% CI 70.2-88.9) and 79.4\% (95\% CI 70-86.5), 
respectively; however, they noted high between-study heterogeneity and lack of precision [24]. This result reflects the insufficiency of PET/CT alone to guide management.

In chest $\mathrm{CT}$, beam hardening artifacts may occur from contrast media in the vessel(s) near the LNs, which tend to cause artifacts in CT morphology, diameter assessment, and CTTA. This may lead to poor measures, and this limitation of CT imaging can be overcome by $\mathrm{LN}$ evaluation using real-time EBUS. There are some established sonographic features suggestive of metastatic LN, including short axis length, round shape, distinct margin, heterogenic echogenicity, and absence of CNS [8-10, 25]. However, previous studies have reported that sonographic features, even after some feature integration, favor metastatic LNs, although with low sensitivity and specificity [25].

Occasionally, small-size LNs, which are not visible on chest $\mathrm{CT}$ or PET/CT, can be observed during EBUS. A dilemma, however, can arise between clinical significance and the risk associated with the procedure as to whether to puncture these small LNs. There is no guidance for these LNs, and the issues of procedural time and specialized personnel are also involved. In this context, there has been an urgent need for an imaging modality that can accurately predict malignant potential. When lung cancer is evaluated in clinical practice, imaging tests, including chest $\mathrm{CT}$ and $\mathrm{PET} / \mathrm{CT}$, are usually performed ahead of EBUS-TBNA. Although LN(s) observed using these image modalities and EBUS are correlated, there is only limited agreement [26]. CTTA has been developed to complement the limitations of existing image modalities, and to enable more accurate malignancy risk prediction.

Digumarthy et al. [14] compared 58 benign and 120 malignant LNs using CTTA; normalized SD had moderate accuracy for differentiating benign LNs from those that were malignant (AUC 0.63 [95\% CI 0.55-0.72]; $P=$ 0.003). Bayanati et al. [27], evaluated 72 LNs from 43 patients, and reported that CTTA had the potential to accurately differentiate malignant and benign mediastinal LNs in lung cancer. They reported that combining six features of CTTA enabled identification of malignant mediastinal nodes with $81 \%$ sensitivity and $80 \%$ specificity (AUC, 0.87; $P<0.0001$ ). Additionally, Andersen et al. [28], reported that mean image intensity values obtained from CTTA, for identification of LNs likely to be malignant, classified $82.6 \%$ of the cases correctly (53\% sensitivity and $97 \%$ specificity) in 46 LNs from 29 patients. The former two studies used TexRAD research software (TexRAD Ltd., Cambridge, United Kingdom), while the latter used MaZda version 4.6 (Institute of Electronics, Technical University of Lodz, Poland). In this study, we used AVIEW (Corelinee Soft, Seoul,
Korea) and compared the diagnostic accuracy with other imaging modalities that are mandatory for staging of lung cancer. The degree of agreement among CTTA software was not studied, nor was itemized standardization achieved.

The important implication of our results is that 2 CTTA parameters-compactness and normalized SDdiffered between benign and malignant LNs. Compactness, which is defined as the ratio of the square of surface area to the total volume of the lesion, is a morphological feature and has been reported to have a higher value in malignant cancers [29, 30], and normalized SD, which is linked to entropy and known to be useful for smaller ROIs, has recently been reported to discriminate malignant from benign LNs [14]. Although normalized SD has a moderate ability to discriminate benign from malignant LNs (AUC, 0.742), it has low clinical utility (sensitivity $8.2 \%$, specificity $100 \%$ ). Moreover, even combining two features in CTTA with PET/CT features, which are known predictors for distinguishing malignant from benign LN, could not achieve better diagnostic accuracy.

In the present study, we sought to identify a parameter to distinguish malignant and benign mediastinal LNs in patients with suspected lung cancer based on pathologically confirmed results by comparing the diagnostic accuracy of various imaging modalities and CTTA. However, there are several important limitations to consider. First, we included a relatively small number of patients and LNs, for whom pathologically proven lymphoma and amyloidosis were excluded from the analysis due to other malignant features different from lung cancer. However, it is difficult to rule out the possibility that this exclusion caused selection bias. Second, it is possible that selection bias was introduced because we analyzed CT images with a thin slice thickness and excluded images acquired from outside hospitals for CTTA. Additionally, there is also the possibility of observer bias, because the segmentation of LNs on CT was performed by a single radiologist. Third, this study was performed in a single institution, and all included EBUS-TBNA was performed by one bronchoscopist with significant experience. Even a small $\mathrm{LN}$ with a short axis diameter of $4 \mathrm{~mm}$ was punctured during EBUS-TBNA, which may be difficult to generalize to other settings. Finally, a standardized CTTA is not yet available for clinical practice and is only being explored for research purposes. During the past few years, many studies have addressed the potential of radiomics. By reviewing these investigations and analyzing data from our study, we agree that the implementation of radiomics in clinical settings remains limited and believe it will be necessary to find other parameters to address its shortcomings. Moreover, we believe that variable background texture 
(i.e., lung, mediastinum, breast) and other factors, such as beam hardening artifact(s), field of view, lesion size and interobserver variability, among others, may affect the results, and that further studies focusing on compensation tools are warranted.

\section{Conclusion}

In this study, we found that some CTTA parameters may help to distinguish between malignant and benign LNs; however, CTTA-guided clinical decisions remain difficult. Therefore, CTTA can only be used as a supplementary aid due to its relatively low diagnostic value. It is necessary to evaluate the clinical significance of LNs by integrating various features on other image modalities because CTTA itself is a novel technique, and further studies are needed.

\section{Additional file}

Additional file 1: Figure S1. Endobronchial ultrasound (EBUS) findings. Round shape (a), oval shape (b), central hilar structure (CHS) (c), homogeneous (d) and heterogeneous (e) echogenicity, and coagulation necrosis sign (CNS) (f). (a) Round shape was defined when the ratio of the short- to long-axis diameter of lymph nodes was $\geq 1.5$ and (b) oval shape was defined as when the ratio was < 1.5. (c) CHS was defined as a linear, flat, hyperechoic area in the center of the lymph node. (d) Distinct margin was defined when the majority of the margin (> 50\%) was clearly visualized with a high echoic border and, if the margin was unclear, the lymph node was assessed with an indistinct margin. (e) Heterogeneous echogenicity was defined as multiple low echoic spots within the lymph node. (f) The CNS is a hypoechoic area within the lymph node without blood flow. (TIFF $850 \mathrm{~kb}$ )

Additional file 2: Table S1. Receiver operating characteristic curve analysis combining features of chest computed tomography (CT), textural features, and 18-fluorodeoxyglucose positron emission tomography/CT (FDG PET/CT) (DOCX 18 kb)

\section{Abbreviations}

AUROC: Area under the receiver operating curve (AUROC);

CART: Classification and regression tree; CNS: Coagulation necrosis sign; CT: Computed tomography; CTTA: Computed tomography texture analysis; EBUS-TBNA: Endobronchial ultrasound-guided transbronchial needle aspiration; FDG: Fluorodeoxyglucose; LN: Lymph node; NSCLC: Non-small-cell lung cancer; PET: Positron emission tomography

\section{Acknowledgements}

SYS was supported by Basic Science Research Program through the National Research Foundation of Korea (NRF) funded by the Ministry of Science, ICT \& Future Planning (NRF-2017R1C1B5076281).

\section{Funding}

This study was not supported by any other sources.

\section{Availability of data and materials}

The datasets used for the current study are available from the corresponding author on reasonable request.

\section{Authors' contributions}

YSJ and SSY planned the study and contributed to the analysis and preparation of the manuscript. YSJ, SSY and IKH contributed to organizing data. YSJ drafted the paper and was responsible for data analysis and interpretation; SSY and ILH critically reviewed the data analysis. All authors approved of the version to be published.

\section{Ethics approval and consent to participate}

The study protocol was approved by the Institutional Review Board of Samsung Medical Center (IRB No., KHUH 2018-08-023).

\section{Consent for publication}

Not required due to study design.

\section{Competing interests}

The authors declare that they have no competing interests.

\section{Publisher's Note}

Springer Nature remains neutral with regard to jurisdictional claims in published maps and institutional affiliations.

\section{Author details}

'Department of Radiology, Kyung Hee University Hospital, College of Medicine, Kyung Hee University, Seoul, Republic of Korea. ${ }^{2}$ Department of Nuclear Medicine, Kyung Hee University Hospital, College of Medicine, Kyung Hee University, Seoul, Republic of Korea. ${ }^{3}$ Division of Pulmonary and Critical Care Medicine, Department of Internal Medicine, KyungHee University Hospital, Seoul, Republic of Korea. ${ }^{4}$ Department of Internal Medicine, Division of Pulmonary, Allergy, and Critical Care Medicine, Hallym University Kangdong Sacred Heart Hospital, Seoul, Republic of Korea.

Received: 14 December 2018 Accepted: 13 May 2019

Published online: 22 May 2019

\section{References}

1. Varela-Lema L, Fernandez-Villar A, Ruano-Ravina A. Effectiveness and safety of endobronchial ultrasound-transbronchial needle aspiration: a systematic review. Eur Respir J. 2009;33:1156-64.

2. Gu P, Zhao YZ, Jiang LY, Zhang W, Xin Y, Han BH. Endobronchial ultrasound-guided transbronchial needle aspiration for staging of lung cancer: a systematic review and meta-analysis. Eur J Cancer. 2009;45: 1389-96.

3. Silvestri GA, Gonzalez AV, Jantz MA, Margolis ML, Gould MK, Tanoue LT, Harris $L$, Detterbeck FC. Methods for staging non-small cell lung cancer: diagnosis and management of lung cancer, 3rd ed: American College of Chest Physicians evidence-based clinical practice guidelines. Chest. 2013; 143:e211S-50S.

4. Yasufuku K, Chiyo M, Koh E, Moriya Y, lyoda A, Sekine Y, Shibuya K, lizasa T, Fujisawa T. Endobronchial ultrasound guided transbronchial needle aspiration for staging of lung cancer. Lung Cancer. 2005:50:347-54.

5. Ernst A, Eberhardt R, Krasnik M, Herth FJ. Efficacy of endobronchial ultrasound-guided transbronchial needle aspiration of hilar lymph nodes for diagnosing and staging cancer. J Thorac Oncol. 2009;4:947-50.

6. Detterbeck FC, Jantz MA, Wallace M, Vansteenkiste J, Silvestri GA: Invasive mediastinal staging of lung cancer: ACCP evidence-based clinical practice guidelines (2nd edition). Chest 2007, 132:202s-220s.

7. Birim O, Kappetein AP, Stijnen T, Bogers AJ. Meta-analysis of positron emission tomographic and computed tomographic imaging in detecting mediastinal lymph node metastases in nonsmall cell lung cancer. Ann Thorac Surg. 2005;79:375-82.

8. Schmid-Bindert G, Jiang H, Kahler G, Saur J, Henzler T, Wang H, Ren S, Zhou C, Pilz LR. Predicting malignancy in mediastinal lymph nodes by endobronchial ultrasound: a new ultrasound scoring system. Respirology. 2012;17:1190-8.

9. Fujiwara T, Yasufuku K, Nakajima T, Chiyo M, Yoshida S, Suzuki M, Shibuya K, Hiroshima K, Nakatani Y, Yoshino I. The utility of sonographic features during endobronchial ultrasound-guided transbronchial needle aspiration for lymph node staging in patients with lung cancer: a standard endobronchial ultrasound image classification system. Chest. 2010;138:6417.

10. Nakajima T, Anayama T, Shingyoji M, Kimura H, Yoshino I, Yasufuku K. Vascular image patterns of lymph nodes for the prediction of metastatic disease during EBUS-TBNA for mediastinal staging of lung cancer. J Thorac Oncol. 2012;7:1009-14.

11. Roberts SA, Mahon BS, Evans R. Coagulation necrosis in malignant mediastinal nodes on endoscopic ultrasound: a new endosonographic sign Clin Radiol. 2005;60:587-91. 
12. Lee N, Inoue K, Yamamoto R, Kinoshita H. Patterns of internal echoes in lymph nodes in the diagnosis of lung cancer metastasis. World J Surg. 1992; 16:986-93 discussion 993-984.

13. Rusch WW, Asamura H, Watanabe H, Giroux DJ, Rami-Porta R, Goldstraw P. The IASLC lung cancer staging project: a proposal for a new international lymph node map in the forthcoming seventh edition of the TNM classification for lung cancer. J Thorac Oncol. 2009:4:568-77.

14. Digumarthy SR, Padole AM, Lo Gullo R, Singh R, Shepard JO, Kalra MK. CT texture analysis of histologically proven benign and malignant lung lesions. Medicine (Baltimore). 2018;97:e11172.

15. Koo HJ, Kim MY, Koo JH, Sung YS, Jung J, Kim SH, Choi CM, Kim HJ. Computerized margin and texture analyses for differentiating bacterial pneumonia and invasive mucinous adenocarcinoma presenting as consolidation. PLoS One. 2017;12:e0177379.

16. Miles KA, Ganeshan B, Hayball MP. CT texture analysis using the filtrationhistogram method: what do the measurements mean? Cancer Imaging. 2013;13:400-6

17. Hoh CK, Hawkins RA, Glaspy JA, Dahlbom M, Tse NY, Hoffman EJ, Schiepers C, Choi Y, Rege S, Nitzsche E, et al. Cancer detection with whole-body PET using 2-[18F]fluoro-2-deoxy-D-glucose. J Comput Assist Tomogr. 1993;17: 582-9.

18. Kim YK, Lee KS, Kim BT, Choi JY, Kim H, Kwon OJ, Shim YM, Yi CA, Kim HY, Chung MJ. Mediastinal nodal staging of nonsmall cell lung cancer using integrated 18F-FDG PET/CT in a tuberculosis-endemic country: diagnostic efficacy in 674 patients. Cancer. 2007;109:1068-77.

19. Shim SS, Lee KS, Kim BT, Chung MJ, Lee EJ, Han J, Choi JY, Kwon OJ, Shim YM, Kim S. Non-small cell lung cancer: prospective comparison of integrated FDG PET/CT and CT alone for preoperative staging. Radiology. 2005;236:1011-9.

20. Kim BT, Lee KS, Shim SS, Choi JY, Kwon OJ, Kim H, Shim YM, Kim J, Kim S. Stage T1 non-small cell lung cancer: preoperative mediastinal nodal staging with integrated FDG PET/CT--a prospective study. Radiology. 2006;241:501-9.

21. Lee JW, Kim EY, Kim DJ, Lee JH, Kang WJ, Lee JD, Yun M. The diagnostic ability of (18)F-FDG PET/CT for mediastinal lymph node staging using (18)FFDG uptake and volumetric $C T$ histogram analysis in non-small cell lung cancer. Eur Radiol. 2016;26:4515-23.

22. Gould MK, Kuschner WG, Rydzak CE, Maclean CC, Demas AN, Shigemitsu H, Chan JK, Owens DK. Test performance of positron emission tomography and computed tomography for mediastinal staging in patients with nonsmall-cell lung cancer: a meta-analysis. Ann Intern Med. 2003;139:879-92.

23. Hellwig D, Graeter TP, Ukena D, Groeschel A, Sybrecht GW, Schaefers HJ, Kirsch CM. 18F-FDG PET for mediastinal staging of lung cancer: which SUV threshold makes sense? J Nucl Med. 2007;48:1761-6.

24. Schmidt-Hansen M, Baldwin DR, Hasler E, Zamora J, Abraira V, Roque IFM: PET-CT for assessing mediastinal lymph node involvement in patients with suspected resectable non-small cell lung cancer. Cochrane Database Syst Rev 2014:Cd009519.

25. Jhun BW, Um SW, Suh GY, Chung MP, Kim H, Kwon OJ, Han J, Lee KJ. Clinical value of endobronchial ultrasound findings for predicting nodal metastasis in patients with suspected lymphadenopathy: a prospective study. J Korean Med Sci. 2014;29:1632-8.

26. Dhooria S, Agarwal R, Aggarwal AN, Gupta N, Gupta D, Behera D. Agreement of mediastinal lymph node size between computed tomography and endobronchial ultrasonography: a study of 617 patients. Ann Thorac Surg. 2015;99:1894-8.

27. Bayanati H, R ET, Souza CA, Sethi-Virmani V, Gupta A, Maziak D, Amjadi K, Dennie C. Quantitative $C T$ texture and shape analysis: can it differentiate benign and malignant mediastinal lymph nodes in patients with primary lung cancer? Eur Radiol. 2015;25:480-7.

28. Andersen MB, Harders SW, Ganeshan B, Thygesen J, Torp Madsen HH, Rasmussen F. CT texture analysis can help differentiate between malignant and benign lymph nodes in the mediastinum in patients suspected for lung cancer. Acta Radiol. 2016;57:669-76.

29. Nie K, Chen JH, Yu HJ, Chu Y, Nalcioglu O, Su MY. Quantitative analysis of lesion morphology and texture features for diagnostic prediction in breast MRI. Acad Radiol. 2008;15:1513-25.

30. Agner SC, Rosen MA, Englander S, Tomaszewski JE, Feldman MD, Zhang P Mies C, Schnall MD, Madabhushi A. Computerized image analysis for identifying triple-negative breast cancers and differentiating them from other molecular subtypes of breast cancer on dynamic contrast-enhanced MR images: a feasibility study. Radiology. 2014;272:91-9.

\section{Ready to submit your research? Choose BMC and benefit from:}

- fast, convenient online submission

- thorough peer review by experienced researchers in your field

- rapid publication on acceptance

- support for research data, including large and complex data types

- gold Open Access which fosters wider collaboration and increased citations

- maximum visibility for your research: over $100 \mathrm{M}$ website views per year

At BMC, research is always in progress.

Learn more biomedcentral.com/submissions 